\title{
Capteur électronique pour la dosimétrie des neutrons
}

\author{
B. BARELAUD, J.L. DECOSSAS, L. MAKOVICKA, J.C. VAREILLE*
}

(Manuscrit reçu le 20 février 1990)

RÉSUMÉ Nous avons étudié la possibilité d'effectuer une dosimétrie neutronique des personnels à l'aide d'un dispositif individuel à microprocesseur, travaillant en temps réel (débitmètre). La plage énergétique à laquelle nous nous intéressons s'étend des neutrons thermiques à $5 \mathrm{MeV}$ environ. Le système est basé, d'une part, sur une méthode différentielle permettant de s'affranchir partiellement des grandeurs interférentes (interactions directes des photons $y$ et des neutrons sur le silicium) et du bruit et, d'autre part, sur un convertisseur de polyéthylène $\left(\mathrm{CH}_{2}\right)_{n}$ implanté par des atomes de bore $\left({ }^{10} \mathrm{~B}\right)$ qui effectue la conversion neutrons-particules chargées secondaires. Ce convertisseur optimisé, en utilisant des calculs de Monte-Carlo, permet d'obtenir une efficacité intrinsèque quasi indépendante de l'énergie des neutrons. La détection est effectuée par des diodes. L'utilisation de la méthode différentielle nous a amenés à l'étude de la symétrie des deux diodes. Nous avons pu montrer qu'un seuil bas d'équivalent de dose de $30 \mu \mathrm{Sv}$ peut être atteint pour les neutrons thermiques et rapides et qu'un débit d'équivalent de dose minimal d'une centaine de $\mu \mathrm{Sv} / \mathrm{h}$ peut être mesuré.

ABSTRACT We studied the possibility of realizing personnel neutron dosimetry using an individual microprocessor controlled device, working in real time (ratemeter). The energy range we are interested in goes from the energy of thermal neutrons up to about $5 \mathrm{MeV}$. The device, based on a differential method, is partially free from interfering signals (interactions of gamma rays and neutrons on silicon) as well as the sources of background. Besides, a boron implanted $\left(\mathrm{CH}_{2}\right)_{n}$ "converter" performs the neutrons to secondary charged particles conversion. The converter, which has been optimized using a Monte Carlo method, shows a dose equivalent response almost independent of neutron energy. The secondary charged particles are detected by diodes. The design of the system led us to study the symetry of the two diodes. We showed that a dose equivalent level as low as $30 \mu \mathrm{Sv}$ can be obtained for thermal and fast neutrons, and that a dose equivalent rate of a hundred $\mu \mathrm{Sv} / \mathrm{h}$ can be measured.

\section{INTRODUCTION}

La dosimétrie des personnels dans les champs de neutrons reste, à l'heure actuelle, un problème encore mal résolu. La plupart des dosimètres existants, basés essentiellement sur la détection de particules secondaires, traitent le problème en analysant les modifications globales des propriétés du matériau sous l'action de ces particules ou par comptage des traces des particules secondaires dans un matériau-cible approprié. Tous ces systèmes (films, émulsions photographiques, détecteurs solides de traces (DST), dosimètres thermoluminescents,...) offrent

" Laboratoire d'électronique des polymères sous faisceau ionique (LEPOFI), Faculté des sciences, 123, av. Albert-Thomas, 87060 Limoges Cedex. 
chacun des avantages tels que simplicité, insensibilité au rayonnement $y_{1}$ faible coût. En contrepartie, ils présentent des inconvénients incontournables : les mesures sont effectuées a posteriori, ils possèdent parfois un bruit de fond important, une reproductibilité difficile à maîtriser ; enfin, on connaît la lourdeur qu'imposent les traitements en dosimétrie individuelle de routine.

Une méthode plus adaptée à l'ère de l'électronique intégrée consiste à faire de la dosimétrie en temps réel. Le principe de la mesure est fondé sur la détection de particules secondaires, et le traitement de l'information s'effectue au rythme de l'acquisition des données. Cela permet d'accéder au fonctionnement en temps réel et, par conséquent, de prévoir aussi une utilisation du dosimètre comme système d'alarme.

Ces dosimètres personnels [7] se décomposent en deux parties bien distinctes et complémentaires : le capteur qui réalise la fonction acquisition, choisi suivant le type de dosimétrie ( $\beta, \gamma$, neutrons), la chaîne électronique qui effectue la fonction traitement en temps réel. Actuellement, pour la dosimétrie neutronique, deux capteurs sont envisageables, le compteur proportionnel $[8-9,20]$ et celui qui fait intervenir un détecteur électronique $[5,25]$.

Pour de multiples raisons, le choix du laboratoire s'est porté sur des diodes au silicium associées à un convertisseur de polyéthylène implanté au bore 10. La principale raison provient du fait que le LEPOFI s'est déjà fortement préoccupé, aussi bien théoriquement qu'expérimentalement, des problèmes de détection des neutrons (thermiques, rapides) à l'aide d'un ensemble convertisseur $\left(\mathrm{CH}_{2}\right)_{n}$ - détecteur type CR 39 [2-4, 13-16, 21-23, 26-27]. De plus, des calculs théoriques approfondis ont été effectués pour caractériser les flux de protons émergeant du convertisseur de $\left(\mathrm{CH}_{2}\right)_{n}$, ces calculs demeurent applicables pour les détecteurs élecroniques.

\section{PRÉSENTATION DU SYSTERGE DE RESURE}

Comme tout système de mesure, notre dispositif a pour but de donner une valeur aussi précise que possible de la ou des grandeurs dosimétriques choisies. Les performances du système de mesure complet sont déduites en combinant de manière appropriée les caractéristiques de transfert. En utilisant un formalisme rigoureux [18], nous pouvons distinguer trois types de sollicitations ou grandeurs d'entrée:

Les mesurandes * ou grandeurs d'entrée désirées (Gd) qui sont les grandeurs pour la mesure desquelles le système est spécifiquement conçu. Dans notre dispositif, cela correspond aux protons émergeant du convertisseur de polyéthylène dus à la diffusion élastique $(n, p)$, ainsi qu'aux particules $\alpha$ et ions ${ }^{7} \mathrm{Li}$ générés par la réaction des neutrons thermiques sur le bore 10.

Les grandeurs interférentes (Gi) qui sont les grandeurs auxquelles le système n'est pas intentionnellement sensible. Elles perturbent la mesure et leurs effets se superposent à celui des grandeurs précédentes à la sortie du système. Les interactions des photons $\gamma$ et des neutrons sur

\footnotetext{
* terme utilisé par les personnes travaillant sur les capteurs et les systèmes de mesure.
} 
le cristal de silicium de la diode sont ces principales grandeurs. Interviennent, à un niveau moindre, le bruit des éléments du dispositif (détecteur, préamplificateur,...), ainsi que le rayonnement naturel.

Les grandeurs modifiantes $(\mathrm{Gm})$ qui sont les grandeurs capables de faire varier la caractéristique de transfert du système aussi bien pour les mesurandes que pour les grandeurs interférentes. Cela correspond à la tension de polarisation de la diode (profondeur de zone désertée), au gain en tension de l'amplificateur, au seuil de discrimination par rapport à la valeur zéro et aux propriétés du convertisseur.

La réponse de notre système est donc composée des grandeurs d'entrée désirées (Gd) d'une part et de celles interférentes (Gi) d'autre part. Toutes deux, soumises à des fluctuations statistiques, peuvent varier en fonction des grandeurs modifiantes. Afin de s'affranchir en partie de la contribution des grandeurs interférentes (Gi), nous avons appliqué la méthode différentielle qui a été proposée par le laboratoire et utilisée par Makovicka [13] avec des détecteurs solides de traces. Le schéma de principe de notre système est présenté sur la figure 1. II comporte deux voies de mesure symétriques en ce qui concerne la chaîne électronique de traitement du signal ; une des voies sert de référence qui n'est sensible qu'aux grandeurs interférentes (détecteu. " sans convertisseur), l'autre voie, qui comporte un détecteur précédé d'un convertisseur, délivre une réponse tributaire de toutes les grandeurs d'entrée. Le signal utile est obtenu en retranchant les réponses de chaque voie et il ne comporte que les impulsions dues aux protons et aux particules $\alpha$ issus du convertisseur et détectés par la diode; il correspond aux mesurandes.

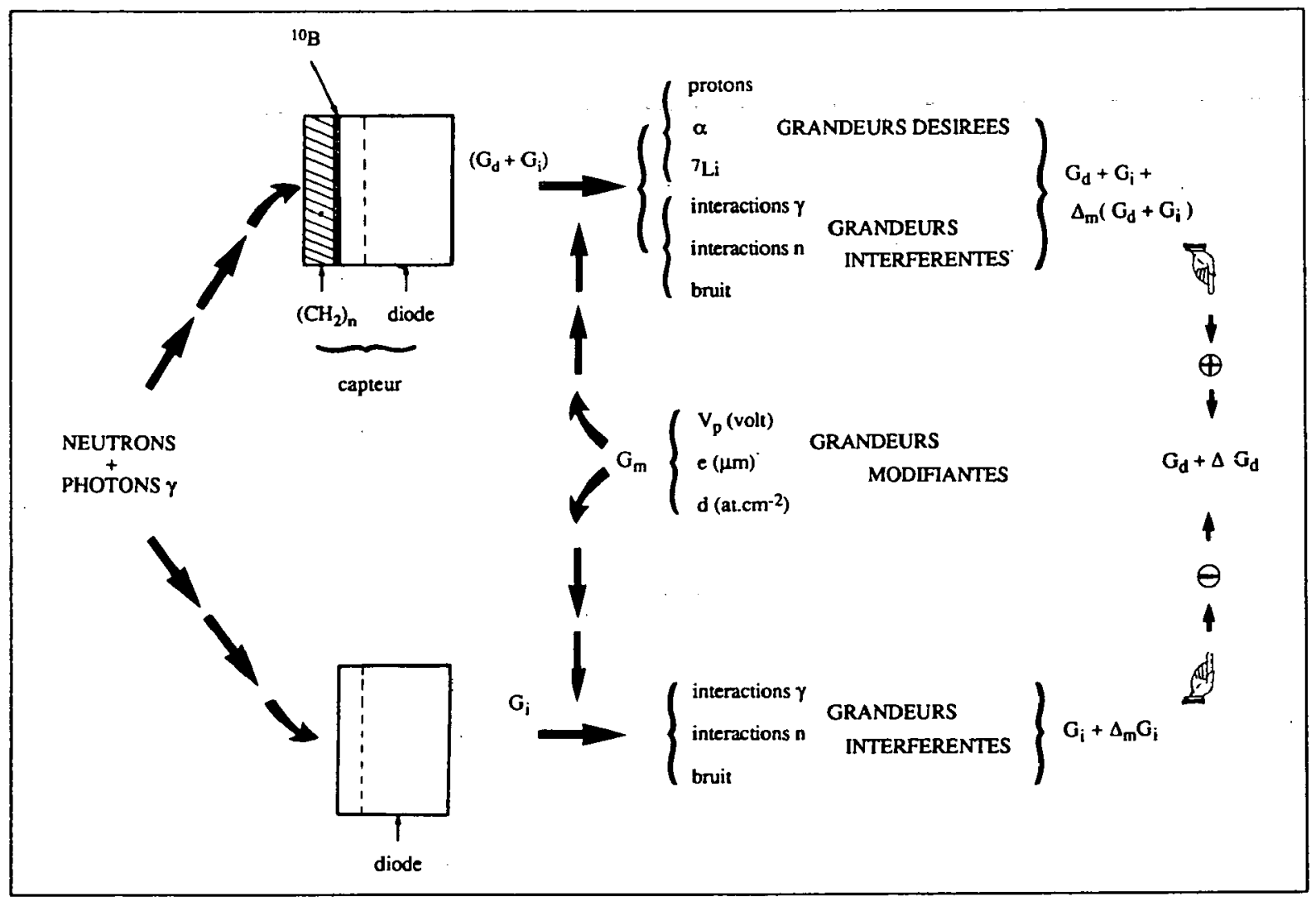

Fig. 1. - Principe du système utlllsé. 


\section{III. ÉTUDE DU CAPTEUR}

Nous désignons sous ce vocable le couple "convertisseur - détecteur" qui est capable de répondre à un faisceau de neutrons.

\section{III.1. Le convertisseur}

Le convertisseur est en polyéthylène implanté au bore 10. II est utilisé sur l'une des deux voies de mesure du dispositif. II délivre:

- des protons qui sont dus à la diffusion élastique des neutrons rapides sur les atomes d'hydrogène du $\left(\mathrm{CH}_{2}\right)_{n}$,

- des particules $\alpha$ et des ions lithium obtenus grâce à la réaction des neutrons (essentiellement thermiques) sur le bore $10\left({ }^{10} \mathrm{~B}(\mathrm{n}, \alpha)^{7} \mathrm{Li}\right)$.

Les calculs théoriques relatifs aux spectres de protons issus du convertisseur [13] fondés sur la méthode Monte-Carlo ont permis d'optimiser la réponse de ce dernier pour les neutrons rapides. En tenant compte du nombre de protons émergeant du convertisseur et de la variation du facteur de conversion d'équivalent de dose $H^{*}(10)$ en fonction de l'énergie et de l'angle d'incidence du faisceau de neutrons, l'efficacité intrinsèque (1000 coups . $\mathrm{cm}^{-2} \cdot \mathrm{mSv}^{-1}$ ) (fig. 2) est quasi-indépendante de l'énergie (de $200 \mathrm{keV}$ à $5 \mathrm{MeV}$ ) et de l'incidence (variation inférieure à $\pm 10 \%)$. Associè à la diode silicium, le convertisseur optimisé à une épaisseur correspondant à $35 \mu \mathrm{m}$.

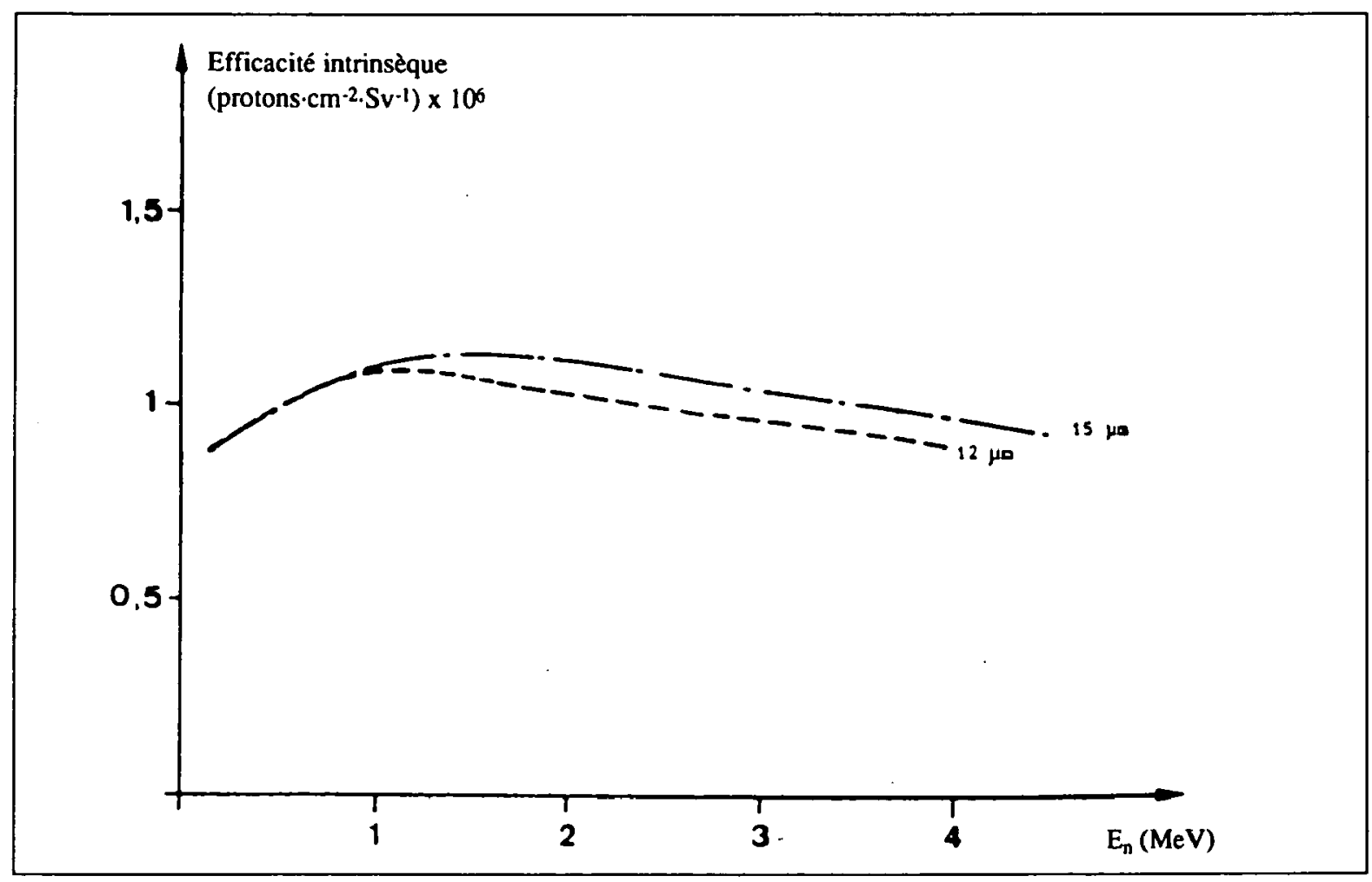

Fig. 2. - Efficacité dosimétrique intrinsèque de convertisseurs $\left(\mathrm{CH}_{2}\right)_{n}$ d'épalsseur de $15 \mu \mathrm{m}$ et $12 \mu \mathrm{m}$ entre 0,2 et $4 \mathrm{MeV}$ pour une incidence aléatolre (Isotrope) des neutrons entre $0^{\circ}$ et $90^{\circ}$. 
On choisit l'efficacité des neutrons thermiques égale à celle des rapides en ajustant la densité d'implantation des atomes de bore 10 dans le polyéthylène (fig. 3). Dans notre cas, l'efficacité intrinsèque de 1000 impulsions $\mathrm{cm}^{-2} \cdot \mathrm{mSv}^{-1}$ est obtenue grâce à une densité de $2,6 \times 10^{15}$ atomes de ${ }^{10} \mathrm{~B} . \mathrm{cm}^{-2}$. La profondeur moyenne d'implantation est égale à $0,3 \mu \mathrm{m}$.

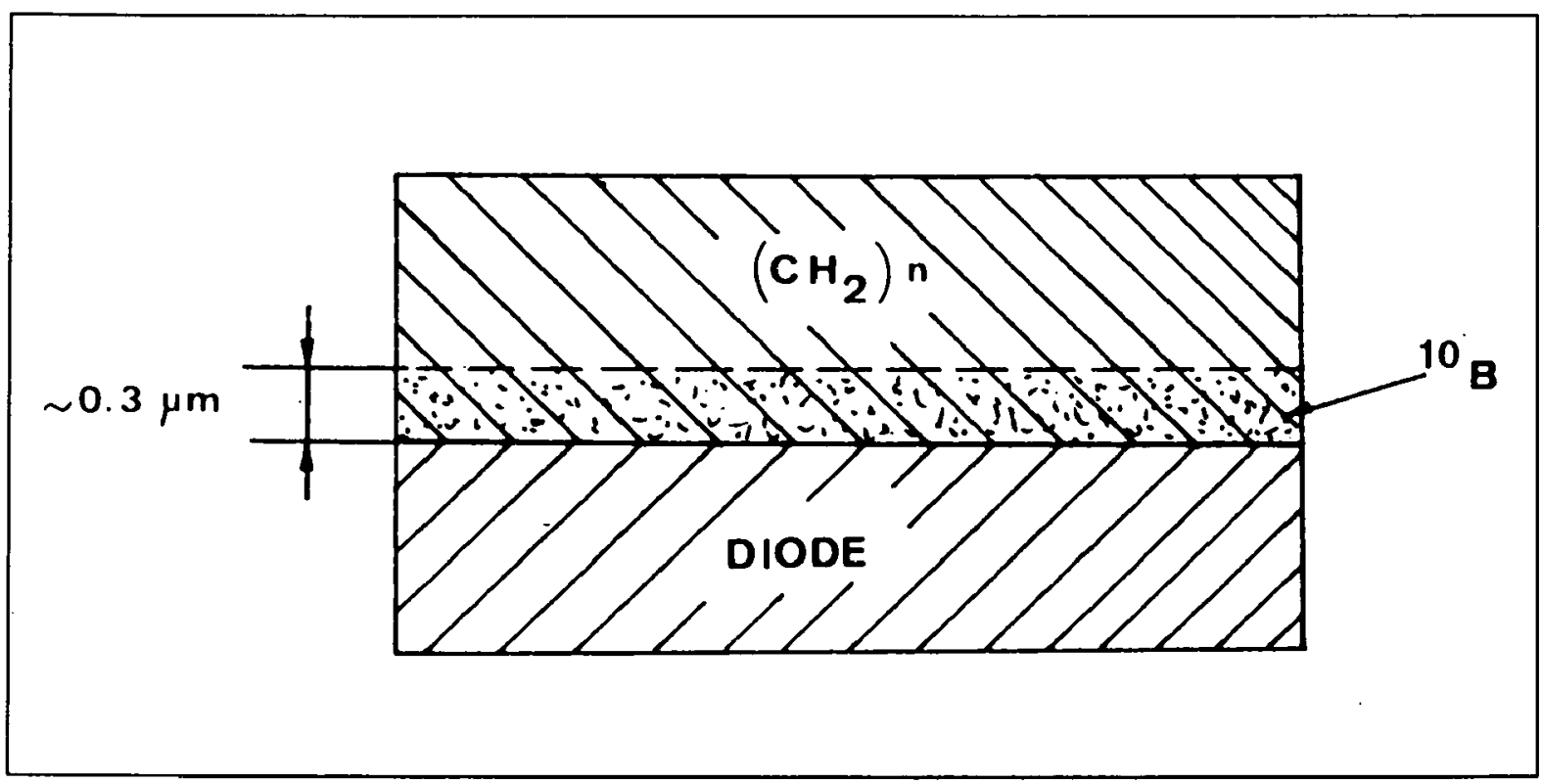

Fig. 3. - Schéma de l'ensemble diode/convertisseur $\left(\mathrm{CH}_{2}\right)_{n}$ avec Implantation de bore 10.

\section{III.2. Les diodes}

Les diodes utilisées sont des jonctions passivées et implantées, le matériau de base étant du silicium de type $N$. Ce processus de fabrication [10-11] permet d'obtenir des diodes avec les propriétés principales suivantes :

- un courant inverse de l'ordre de 1 à $10 \mathrm{nA} / \mathrm{cm}^{2}$ pour une zone désertée de $100 \mathrm{jm}$;

- une épaisseur de fenêtre d'entrée d'environ $2000 \AA$ équivalent Si ;

- une excellente résolution en énergie.

Certaines caractéristiques des diodes fabriquées par la Société ENERTEC ont été modifiées pour répondre à nos besoins. Notre choix s'est porté sur des diodes partiellement désertées qui sont adaptées à la spectrométrie des particules chargées, de surface $150 \mathrm{~mm}^{2}$. Elles se caractérisent par:

- une tension de polarisation faible $\left(V_{\max }=9 \mathrm{~V}\right)$ susceptible d'être fournie par pile et, surtout, un fonctionnement à la pression atmosphérique ; 
- une zone $P$ réalisée par implantation de bore dans le silicium, le bore 11 a été utilisé pour éviter les réactions $(n, \alpha)$ sur le bore 10 qui, ici, seraient apparues comme parasites;

- une profondeur de zone désertée de $100 \mu \mathrm{m}$ à $9 \mathrm{~V}$ qui permet aux particules $\alpha$ de $12 \mathrm{MeV}$ et aux protons de 3,5 MeV de déposer toute leur énergie dans le silicium; cette zone peut éventuellement être modifiée en jouant sur la tension de polarisation, avec un double effet:

- les plus énergétiques des protons risquent de ne pas déposer toute leur énergie dans la zone désertée, ce qui peut constituer un handicap pour un système de spectrométrie mais ne gêne en rien un dosimètre basé sur le simple comptage des particules;

- les interactions parasites, essentiellement dues aux photons $\gamma$, sources d'impulsions de bruit, devraient.ainsi pouvoir être réduites;

- la fenêtre d'entrée la plus fine possible (1500 Å équivalent silicium) afin de réduire la perte d'énergie dans cette zone;

- le contact avant en or qui est un matériau avec une section efficace d'interaction relativement faible aux neutrons rapides; la probabilité d'interaction des neutrons thermiques est minimisée de par la très faible épaisseur du dépôt d'or (les $1500 \AA$ correspondant au dépôt d'or et à l'épaisseur de l'oxyde de silicium) ;

- les matériaux employés pour la structure de la diode qui sont dits "bas bruit" pour nous permettre de diminuer la moyenne du nombre de coups parasites par heure;

- une symétrisation des diodes la meilleure possible.

Notre système étant fondé sur l'application de la méthode différentielle; il est donc nécessaire que les deux voies de mesure soient symétriques. La partie électronique analogique et numérique du système peut, à l'aide de réglages minutieux, être mise au point correctement. Le point crucial concerne les diodes qui, technologiquement, ne peuvent pas être rigoureusement identiques.

Dans notre cas, l'étude de la variation de la profondeur de zone désertée $\mathcal{X}$ et du courant inverse en fonction de la tension des deux diodes permet de mettre en évidence leur dissymétrie (fig. 4). De plus, les bruits engendrés par les diodes ne sont pas similaires. L'étude de ces trois paramètres montre une dissymétrie fort génante que le constructeur n'a apparemment pas su surmonter. Cela a constitué une difficulté pour notre travail, mais nul doute que l'on pourra améliorer nettement la symétrie des diodes utilisées. Cependant, en l'état, les réponses obtenues sur chacune des voies ne peuvent plus être utilisées directement (soustraction) pour délivrer la réponse désirèe. Ce problème, d'ordre technologique, va nous priver de l'utilisation simple de cette méthode dans certaines de nos expérimentations. 


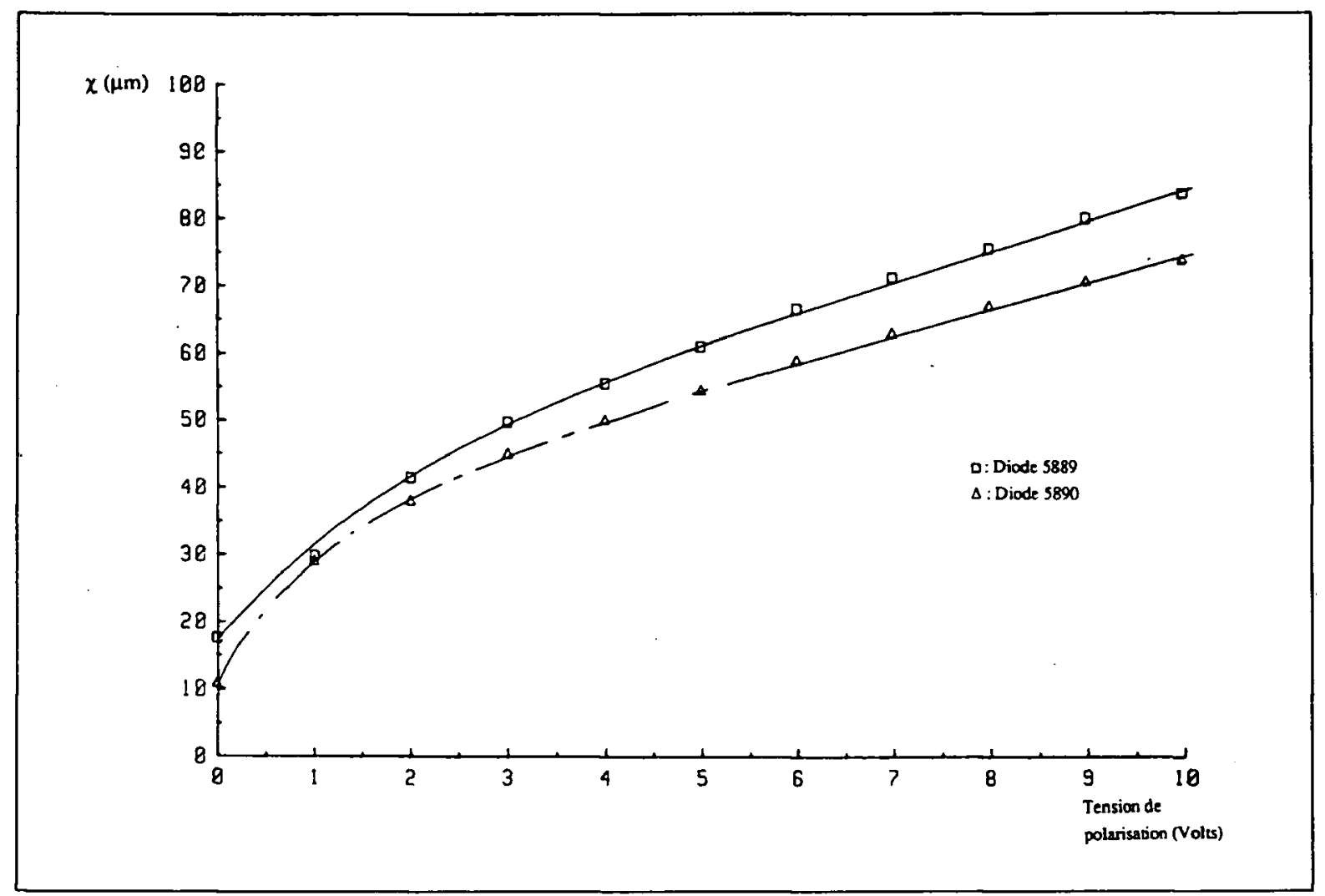

Flg. 4. - Courbe donnant la profondeur de zone désertée $\mathcal{X}$ en $\mu \mathrm{m}$ en fonction de la tension de polarisation.

\section{DESCRIPTION DE LA CHAINE D'ACQUISITION}

Nous allons passer en revue l'une des voies de mesure du dispositif, ces dernières étant symétriques.

Le capteur est relié directement à un préamplificateur de charge qui permet d'intégrer et de mettre en forme les impulsions. Cet élément qui subit une contre-réaction par une capacité dite "de mesure" assure la conversion charge-tension. Les impulsions issues de ce préamplificateur sont amplifiées linéairement et mises en forme par différenciation et intégration. Un circuit restaurateur de ligne de base, la compensation pôlezéro et l'ajustement de la tension continue de sortie à zéro sont prévus au niveau de l'amplificateur afin d'obtenir une bonne résolution. Le traitement des impulsions, issues de l'amplificateur, est effectué à l'aide d'une carte intégrable dans un IBM PC ou compatible. Cette carte correspond à un analyseur multicanal dont la gestion est effectuée par un logiciel approprié.

Le problème majeur est de pouvoir se fixer un seuil énergétique le plus bas possible pour arriver à discriminer les impulsions dues aux bruits intrinsèques de la chaîne et aux divers rayonnements $(\gamma \ldots$... de celles qui proviennent de la contribution des particules secondaires détectées.

L'analyse des résultats est effectuée grâce à des méthodes statistiques. 


\section{LES GRANDEURS INTERFÉRENTES ET LE BRUIT}

\section{V.1. Le bruit}

Nous définissons le bruit comme le nombre d'impulsions enregistrées par unité de temps dues, d'une part, au détecteur polarisé qui n'est soumis qu'au rayonnement naturel ambiant et d'autre part, à l'ensemble des bruits de la chaîne de traitement du signal.

Plusieurs expériences ont été mises en œuvre pour tester la validité des différents réglages et apprécier leur optimisation sur certains résultats. Des séries de mesures réalisées sur $2 \mathrm{~h}$ de temps d'acquisition nous ont donné les résultats suivants (tableau l):

TABLEAU I

Bruit intrinsèque classé dans les différents canaux après optimisation du système

\begin{tabular}{|c|c|c|c|c|c|c|}
\hline $\begin{array}{l}\text { Expe } \\
\text { fience } \\
\tilde{n}^{6}\end{array}$ & 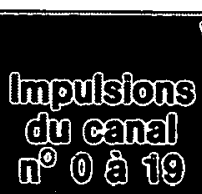 & 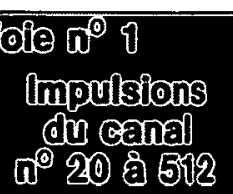 & $\begin{array}{l}\text { topoufistons } \\
\text { totales }\end{array}$ & 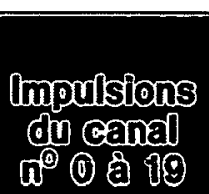 & 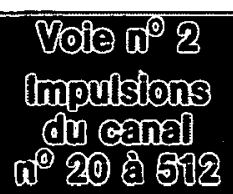 & $\begin{array}{c}\text { loppulsions } \\
\text { cotales }\end{array}$ \\
\hline 1 & 179 & 29 & 208 & 202 & 18 & 220 \\
\hline 2 & 209 & 24 & 233 & 222 & 21 & 243 \\
\hline 3 & 166 & 26 & 192 & 200 & 24 & 224 \\
\hline 4 & 135 & 27 & 162 & 209 & 21 & 230 \\
\hline 5 & 148 & 36 & 184 & 172 & 20 & 192 \\
\hline 6 & 146 & 32 & 178 & 162 & 21 & 183 \\
\hline 7 & 155 & 16 & 171 & 189 & 18 & 207 \\
\hline 8 & 154 & 30 & 184 & 192 & 21 & 213 \\
\hline 9 & 144 & 37 & 181 & 197 & 23 & 220 \\
\hline 10 & 145 & 25 & 170 & 177 & 35 & 212 \\
\hline $\mathrm{m}$ & 158 & 28 & 186 & 192 & 22 & 214 \\
\hline
\end{tabular}

Nous constatons tout d'abord que la majeure partie des impulsions enregistrées se localise dans les vingt premiers canaux, le canal 20 correspondant à une énergie de $389 \mathrm{keV}$. La deuxième constatation concerne la dissymétrie qui existe entre les deux voies bien que la chaîne d'acquisition ait été optimisée. Ces résultats nous donnent une évaluation du bruit qui se situe aux alentours d'une dizaine d'impulsions par heure pour une gamme d'énergie limitée par un seuil bas de $400 \mathrm{keV}$.

\section{V.2. Grandeurs interférentes}

a) Les rayonnements $\gamma$

Dans le cas du silicium, et pour les énergies qui nous concernent, les mécanismes d'interaction principaux (effets photoélectrique, Compton, matérialisation) suscitent l'émission d'un ou de plusieurs électrons 
rapides, dont l'effet ionisant sur la matière permet de détecter la présence des photons. Dans la mesure où la dosimétrie neutronique envisagée est une corrélation simple entre l'équivalent de dose du flux neutronique et le nombre d'impulsions dues aux protons et aux particules $\alpha$, les impulsions provoquées par les $y$ sont parasites, et constituent donc une contribution à minimiser, voire à éliminer. On sait que l'énergie transférée à un électron Compton varie avec l'angle sous lequel l'électron est projeté. L'énergie transférée est maximale pour un "choc" frontal et son rapport avec l'énergie du photon est donné par le tableau II.

\section{TABLEAU}

Fraction de l'énergie transmise à un électron Compton dans un choc frontal avec un photon $\gamma$ d'énergie E, d'après [24]

\begin{tabular}{|cccccccccc|}
\hline$E(\mathrm{MeV})$ & 0.2 & 0.4 & 0.6 & 0.8 & 1 & 1.5 & 2 & 2.5 & 3 \\
\hline$E_{\mathrm{a} \max } / \mathrm{E}$ & 0,44 & 0,61 & 0,70 & 0,76 & 0,80 & 0,85 & 0,89 & 0,91 & 0,92 \\
\hline
\end{tabular}

On constate que des impulsions parasites dues aux photons $y$ peuvent avoir une amplitude importante et constituer un handicap pour la dosimétrie neutronique. Pour chiffrer, dans un exemple concret, l'action des photons y sur les diodes, nous avons réalisé une étude expérimentale.

\section{* Contrôles expérimentaux de nos diodes silicium sous $\gamma$}

La diode utilisée a été exposée à une distance de $0,2 \mathrm{~m}$ à une source de ${ }^{60} \mathrm{Co}$ d'activité égale à $1,86 \cdot 10^{5} \mathrm{~Bq}$. Les résultats que nous donnons correspondent aux valeurs moyennes de cinq mesures effectuées pour deux profondeurs de zone désertée (tableau III et fig. 5) et en tenant compte du seuil situé au canal $\mathrm{n}^{\circ} 20(\mathrm{E} \approx 390 \mathrm{keV})$.

\section{TABLEAU III}

Réponse d'une diode au silicium sous un rayonnement gamma

\begin{tabular}{|lcc|}
\hline Tension de polarisation de la diode $(\mathrm{V})$ & 8 & 3 \\
\hline Profondeur de zone désertée $(\mu \mathrm{m})$ & 75 & 50 \\
\hline $\begin{array}{l}\text { Bruit intrinsèque (impulsions } \cdot \mathrm{cm}^{-2} \cdot \mathrm{h}^{-1} \text { ) } \\
\text { (sans source) }\end{array}$ & 28 & 26 \\
\hline $\begin{array}{l}\text { Réponse de la diode en présence } \\
\left.\text { de la source (impulsions } . \mathrm{cm}^{-2} \cdot \mathrm{h}^{-1}\right)\end{array}$ & 111 & 33 \\
\hline $\begin{array}{l}\text { Contribution de la source } \\
\left.\text { (impulsions } \cdot \mathrm{cm}^{-2} \cdot \mathrm{h}^{-1}\right)\end{array}$ & 83 & 7 \\
\hline \hline Energie maximale du spectre $(\mathrm{keV})$ & 560 & 450 \\
\hline
\end{tabular}




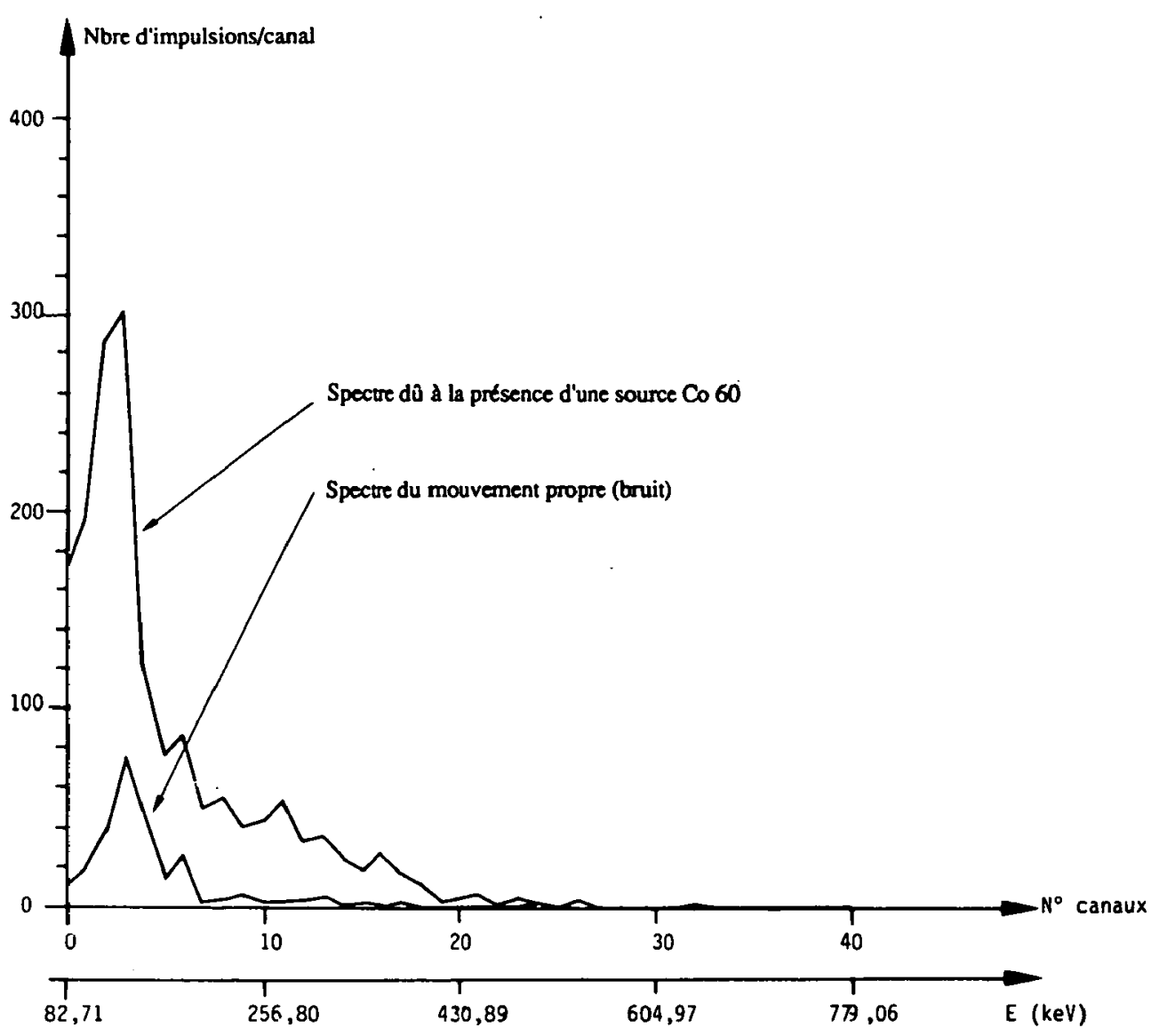

Fig. 5. - Spectre énergétlque de la réponse au rayoǹnement $\gamma$ du cobalt 60, $W=50 \mu \mathrm{m}$.

A partir de ces résultats, nous avons calculé la réponse aux photons $\gamma$ en termes d'impulsions $\mathrm{cm}^{-2} \cdot \mathrm{mSv}^{-1}$. D'après G.C. Messenger [17], l'énergie déposée pour la fluence unité pour une source de ${ }^{60}$ Co est de 4,48 pGy/photon $\mathrm{y} / \mathrm{cm}^{2}$ dans un milieu équivalent au tissu. Dans nos conditions expérimentales, en prenant comme valeur du facteur de qualité effectif moyen (ICRU 40) $\bar{Q}_{\text {eff }}=0,5$ nous pouvons donner. un débit d'équivalent de dose d'environ : $0,6 \mu \mathrm{Sv} / \mathrm{h}$, et donc la réponse en nombre d'impulsions $\cdot \mathrm{cm}^{-2} \cdot \mathrm{mSv}^{-1}$, soit :

$-138 \times 10^{3}$ impulsions. $\mathrm{cm}^{-2} \mathrm{mSv}^{-1}$ pour $\mathrm{W}=75 \mu \mathrm{m}$

$-11,7 \times 10^{3}$ impulsions . $\mathrm{cm}^{-2} \mathrm{mSv}^{-1}$ pour $\mathrm{W}=50 \mu \mathrm{m}$.

De plus, nous constatons l'influence de la profondeur de zone désertée sur la réponse "parasite" due aux photons $\gamma$. Il paraît souhaitable de réduire autant que faire se peut cette profondeur afin de minimiser le volume sensible de la diode. Cela aura pour conséquences de réduire le nombre d'impulsions dues aux photons $y$ ainsi que de limiter en énergie le spectre de leur réponse. 


\section{b) Action directe des neutrons sur le silicium}

L'interaction directe des neutrons avec un détecteur semiconducteur au silicium peut se produire de diverses manières. Deux cas bien distincts peuvent être mis en évidence. Le premier correspond aux interactions qui ont lieu dans la zone sensible du détecteur; elles donnent naissance à des impulsions parasites. Le deuxième cas est l'action des neutrons hors de cette zone, c'est-à-dire sur le boîtier, la colle d'assemblage des divers éléments; cela n'interviendra quasiment pas dans notre dispositif. De plus, nous pouvons négliger les éventuelles interactions avec la couche d'or de métallisation de la face avant à cause de sa faible épaisseur (1500 $\AA$ équivalent silicium). Nous nous limiterons donc aux interactions qui se produisent dans la zone désertée et qui sont principalement des collisions élastiques avec les noyaux de silicium. L'énergie gagnée par le noyau de recul est donnée par la relation suivante [12] :

$$
E_{S i}=\frac{4 A}{(1+A)^{2}}\left(\cos ^{2} \theta\right) E_{n}
$$

A : rapport de la masse du noyau-cible sur la masse du neutron,

$E_{n}$ : énergie du neutron incident,

$E_{\mathrm{si}}$ : énergie du noyau de recul,

$\Theta$ : angle de diffusion du noyau de recul dans le système du laboratoire.

$E_{\mathrm{si}}$ sera maximal pour $\Theta=0$; dans ce cas, l'énergie transmise à un atome de silicium par un neutron est environ de $1 / 7 \mathrm{E}_{\mathrm{n}}$ et comme la section efficace de diffusion élastique est de l'ordre de quelques barns, la contribution de cette interaction est bien à prendre en compte pour les neutrons de la gamme d'énergie la plus élevée.

De plus, certaines réactions nucléaires $\left[(n, \alpha)\right.$ et $(n, p)$ sur ${ }^{29} \mathrm{Si}$ et $\left.{ }^{30} \mathrm{Si}\right]$, pour des neutrons d'énergies supérieures à $E>7 \mathrm{MeV}$, peuvent se produire car elles présentent une section efficace relativement importante.

Ainsi l'interaction directe des neutrons avec le cristal de silicium contribue aux grandeurs interférentes aux énergies élevées.

\section{CALCULS ET RÉSULTATS EXPÉRIMENTAUX}

Ce paragraphe consacré à l'étude du comportement de notre dispositif sous divers champs de neutrons, est structuré de telle sorte que l'on puisse effectuer des comparaisons, d'une part, entre les résultats théoriques et expérimentaux et, d'autre part, entre les résultats d'un ensemble (convertisseur $\left(\mathrm{CH}_{2}\right)_{n}$ - détecteur solide de traces $\mathrm{CR}$ 39) et ceux de notre système de mesure électronique. Diverses caractéristiques sont mises en évidence de manière à situer la qualité des réponses obtenues, 
ainsi que la validité du dispositif. Notre travail expérimental est divisé en deux parties, l'une correspondant aux neutrons rapides et l'autre aux neutrons thermiques.

Le tableau IV résume brièvement les divers champs de mesure.

TABLEAU IV

Classification des champs d'expérimentation

\begin{tabular}{|llll|}
\hline Classe énergétique & Caractéristique du champ & $\begin{array}{l}\text { Equivalent de dose } \\
\text { Débit de dose }\end{array}$ & Site \\
\hline \hline Neutrons rapides & $\begin{array}{l}\text { Flux monoénergétique } \\
(3,3 \mathrm{MeV}) \text { en incidence } \\
\text { normale }\end{array}$ & $\begin{array}{l}5 \mathrm{mSv}, 10 \mathrm{mSv} \text { et } \\
15 \mathrm{mSv}- \\
0,23 \mathrm{~Sv} / \mathrm{h}\end{array}$ & $\begin{array}{l}\text { CEN } \\
\text { Fontenay- } \\
\text { aux-Roses }\end{array}$ \\
\hline Source Am-Be & $\begin{array}{l}\text { Spectre continu } \\
\text { de } 100 \mathrm{keV} \\
\text { à } 11,2 \mathrm{MeV}\end{array}$ & $\begin{array}{l}\text { Mesure effectuée } \\
\text { sur } 1 \mathrm{~h} \\
\text { Débit de dose estimé } \\
\text { à 1,19 mSv/h }\end{array}$ & $\begin{array}{l}\text { LEPOFI } \\
\text { Limoges }\end{array}$ \\
\hline \hline Neutrons thermiques & $\mathrm{E}_{\mathrm{Nth}} \sim 0,025 \mathrm{eV}$ & $\begin{array}{l}\text { Mesure effectuée } \\
\text { sur } 1 \mathrm{~h} \\
\text { Débit : } 180 \mu \mathrm{Sv} / \mathrm{h}\end{array}$ & $\begin{array}{l}\text { CEN } \\
\text { Cadarache }\end{array}$ \\
\hline
\end{tabular}

\section{VI.1. Réponse à un flux de neutrons rapides monoénergétiques sous incidence normale}

Les mesures ont été réalisées sous trois équivalents de dose $(5,10$ et $15 \mathrm{mSv})$ avec trois épaisseurs de convertisseurs $(20 \mu \mathrm{m}, 35 \mu \mathrm{m}$ et $150 \mu \mathrm{m})$. Pour un équivalent de dose donné et une épaisseur de convertisseur déterminée, chaque mesure a été répétée quatre ou cinq fois. La profondeur de la zone désertée des diodes a été déterminée de telle façon que les protons de recul d'énergie maximale $\left(E_{n}=3,3 \mathrm{MeV}\right)$ déposent toute leur énergie.

\section{A - Résultats}

Les résultats sont donnés sous forme de courbes et de tableaux afin de les analyser et de tirer des conclusions. Comme nous allons le voir, ils sont comparables, dans une certaine mesure, à ceux obtenus au laboratoire par D. Paul et L. Makovicka $[19,13]$ avec des DST (CR 39). Nous donnons un exemple précis de spectres bruts obtenus avec des neutrons de 3,4 MeV, $15 \mathrm{mSv}$ et après application de la méthode différentielle (épaisseur du convertisseur $35 \mu \mathrm{m}$, tension diode $10 \mathrm{~V}$ ) (fig. 6).

Ces spectres expérimentaux nous ont permis de vérifier les calculs théoriques effectués sur le convertisseur. 

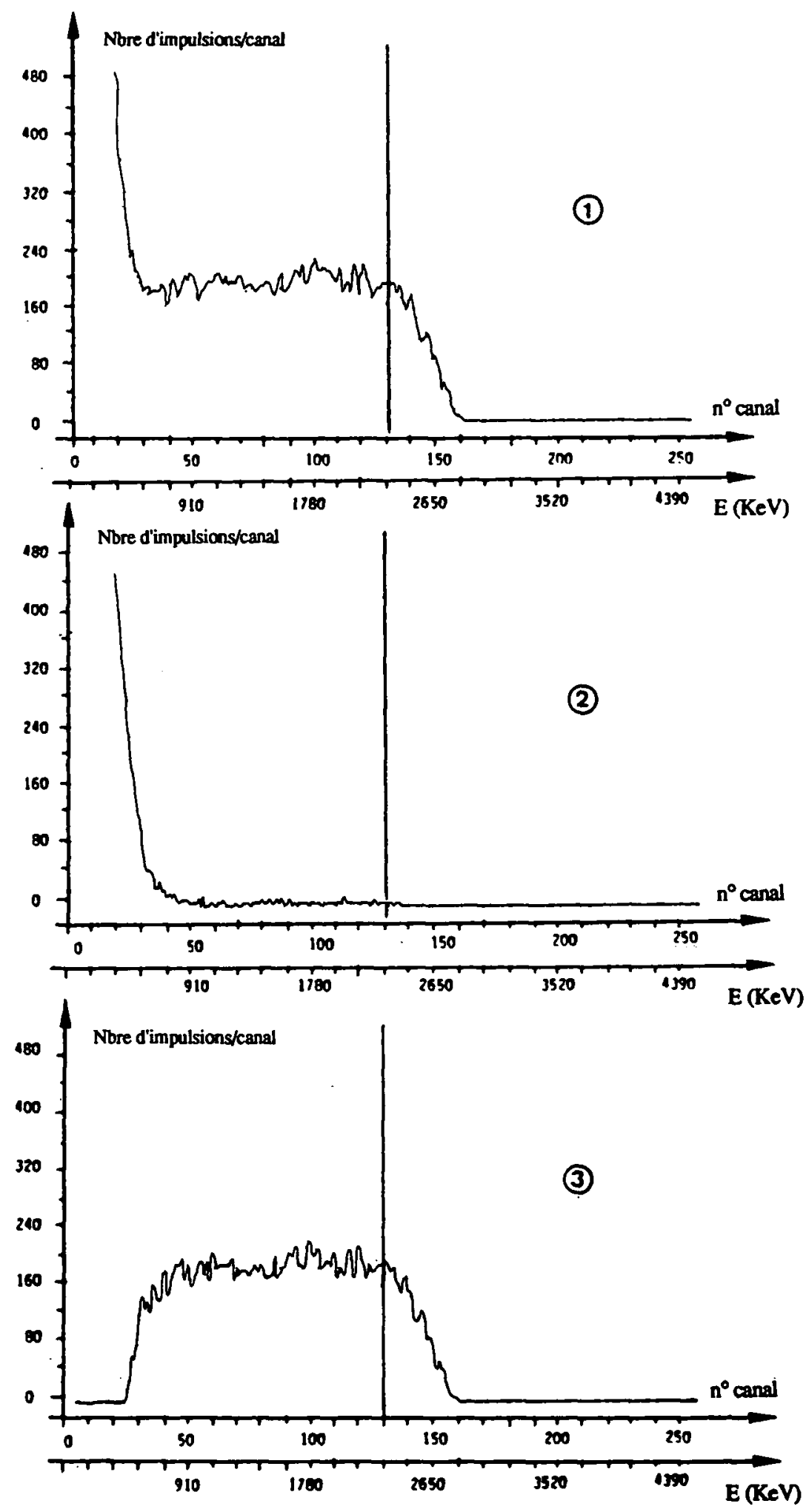

Fig. 6. - Spectres énergétlques enreglstrés des protons lssus du convertlsseur (neutrons d'énergle 3,4 MeV, equivalent de dose $15 \mathrm{mSv}$, épalsseur du convertlsseur $35 \mu \mathrm{m}$, tension de polarlsation $10 \mathrm{~V}$, spectre $n^{\circ} 1$ : convertlsseur + détecteur, spectre $n^{\circ} 2$ : détecteur seul, spectre $n^{\circ} 3$ : résultat de la méthode difrérentlelle). 
En deçà d'un seuil situé environ au canal $n^{\circ} 20$ ( $\left.E \approx 390 \mathrm{keV}\right)$, il ne nous est pas possible, actuellement, d'extraire le signal utile du signal global. En effet, dans cette plage (canal $n^{\circ} 0$ à $n^{\circ} 20$ ) le bruit est prépondérant par rapport au signal attendu, l'origine de ce bruit perturbateur pouvant être lié aux rayonnements $\gamma$ dont nous avons montré l'influence. La perte d'information dans cette région, sans être chiffrée exactement, peut être estimée, d'après les calculs théoriques que nous avons effectués, à environ 10 ou $20 \%$. Les résultats obtenus au cours des diverses mesures sont résumés dans le tableau $\mathrm{V}$.

TABLEAU $V$

Résultats obtenus par des irradiations en neutrons d'énergie 3,3 MeV, en incidence normale

\begin{tabular}{|c|c|c|c|c|c|c|c|}
\hline $\begin{array}{l}\text { Abre de } \\
\text { mesures } \\
\end{array}$ & $\begin{array}{l}\text { Tension do } \\
\text { polarisafion } \\
\text { des dlodes } \\
\text { (volts) }\end{array}$ & $\begin{array}{l}\text { Equivalents } \\
\text { de dose } \\
\text { (mSv) }\end{array}$ & 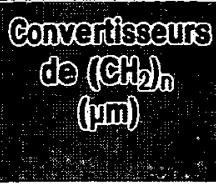 & 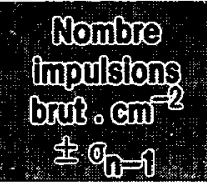 & $\begin{array}{l}\text { Cmpulsions } \\
\text { de brufit } \\
\left(\mathrm{cm}^{-2}\right)^{2} \\
\text { A }_{\mathrm{H}=\mathrm{d}}\end{array}$ & 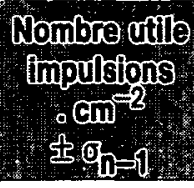 & $\begin{array}{l}\text { Reponse en } \\
\text { timpulsons } \\
\mathrm{Cm}^{-2} \mathrm{zmSy}\end{array}$ \\
\hline 5 & 10 & 5 & 20 & $3053 \pm 107$ & $882 \pm 46$ & $2171 \pm 116$ & $434 \pm 23$ \\
\hline 5 & 10 & 5 & 35 & $5330 \pm 100$ & $1001 \pm 18$ & $4329 \pm 102$ & $866 \pm 20$ \\
\hline 5 & 10 & 5 & 150 & $6116 \pm 311$ & $926 \pm 68$ & $5190 \pm 318$ & $1038 \pm 64$ \\
\hline 5 & 10 & 10 & 20 & $6209 \pm 145$ & $1817 \pm 58$ & $4392 \pm 156$ & $439 \pm 16$ \\
\hline 7 & 10 & 10 & 35 & $11285 \pm 446$ & $1629 \pm 76$ & $9656 \pm 452$ & $966 \pm 45$ \\
\hline 6 & 10 & 10 & 150 & $18966 \pm 734$ & $1564 \pm 50$ & $17402 \pm 736$ & $1740 \pm 74$ \\
\hline 5 & 10 & 15 & 20 & $9711 \pm 200$ & $2396 \pm 286$ & $7315 \pm 349$ & $488 \pm 23$ \\
\hline 4 & 10 & 15 & 35 & $15976 \pm 103$ & $2094 \pm 37$ & $13882 \pm 109$ & $925 \pm 7$ \\
\hline 4 & 10 & 15 & 150 & $28853 \pm 469$ & $1934 \pm 38$ & $26920 \pm 470$ & $1795 \pm 31$ \\
\hline 4 & 6 & 15 & 20 & $9410 \pm 121$ & $1067 \pm 30$ & $8343 \pm 125$ & $556 \pm 8$ \\
\hline 4 & 6 & 15 & 35 & $\$ 1$ & $992 \pm 30$ & $14359 \pm 144$ & $957 \pm 10$ \\
\hline 4 & 6 & 15 & 150 & $28160 \pm 318$ & $871 \pm 19$ & $27290 \pm 319$ & $1819 \pm 21$ \\
\hline
\end{tabular}

$\sigma_{n-1}$ représentant l'écart quadratique moyen, nous donne une indication sur les limites du système en ce qui concerne les faibles doses.

\section{$B$ - Détermination des efficacités et discussion}

Les résultats qui vont suivre sont directement comparables à ceux obtenus au LEPOFI à l'aide des DST, les expériences ayant été menées en parallèle [19].

La dosimétrie neutronique repose sur l'efficacité effective (impulsions . $\mathrm{cm}^{-2} . \mathrm{mSv}^{-1}$ ) du dosimètre ; elle permet d'obtenir la correspondance entre le nombre d'impulsions . $\mathrm{cm}^{-2}$ et l'équivalent de dose (fig. 7). Les droites en pointillés sont obtenues par un programme de régression linéaire dans lequel les points expérimentaux et l'origine sont pris en compte. La pente de ces droites conduit à l'efficacité effective. On constate qu'avec les diodes (fig. 8) les efficacités sont beaucoup plus élevées qu'avec les DST (plus du double) et elles sont d'ailleurs très proches des efficacités intrinsèques du convertisseur (dans le cas d'un convertisseur d'épaisseur $35 \mu \mathrm{m}$, elle est égale à 918 protons $. \mathrm{cm}^{-2} \times \mathrm{mSv}^{-1}$ ). II apparaît donc à l'évidence que les limitations à la détection des neutrons par nos détecteurs électroniques sont très largement inférieures à celles des DST. 
CAPTEUR ÉLECTRONIQUE POUR LA DOSIMÉTRIE DES NEUTR'ONS

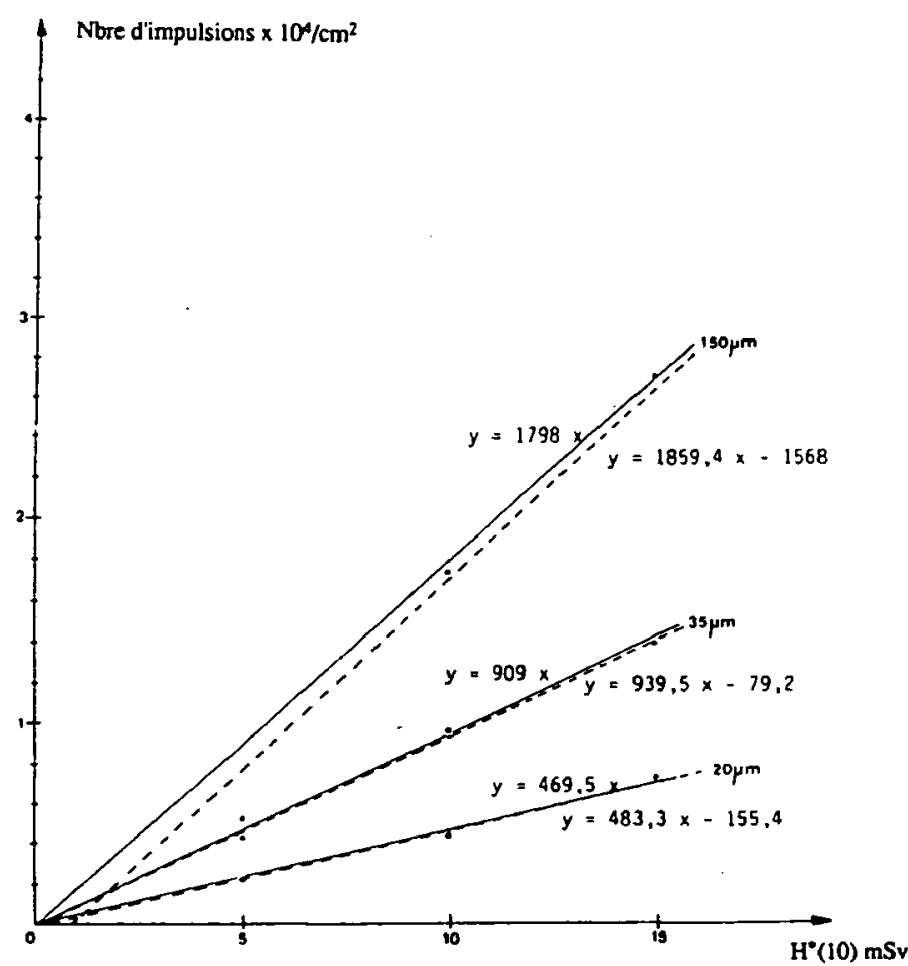

Fig. 7. - Efficacité effective du dosimètre électronique (mesure différentielle) en fonction de l'équivalent de dose à 3,3 MeV pour diverses épaisseurs de convertisseur.

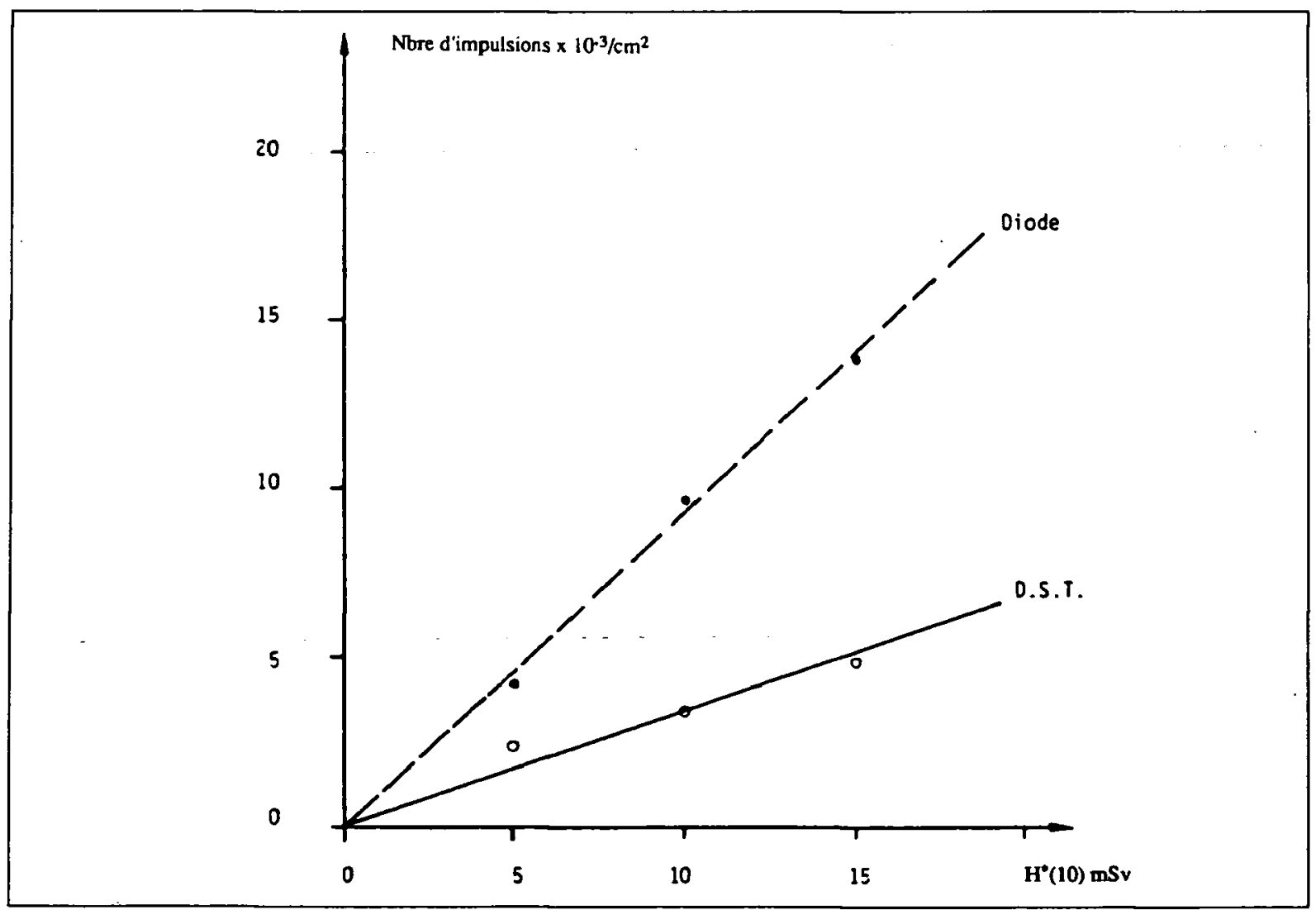

Fig. 8. - Comparalson des efficacités effective obtenues à l'alde des dos/mètres à DST et à diodes, convertisseur de $35 \mu \mathrm{m}$ d'épalsseur. 


\section{VI.2. Comportement du dispositif soumis à une source de neutrons Am-Be}

Comme nous venons de le voir, il existe un bon accord entre les résultats expérimentaux et les calculs à $3,3 \mathrm{MeV}$. Les résultats théoriques sur le convertisseur ( $\$$ III-1) prévoient que l'efficacité est constante à environ $10 \%$ près pour la gamme $200 \mathrm{keV}$ - $5 \mathrm{MeV}$, bien que les vérifications expérimentales correspondantes n'aient pas à ce jour encore été effectuées. Nous avons admis ce résultat pour calculer la réponse du système dans un spectre neutronique $(\mathrm{Am}-\mathrm{Be})$ et la comparer à l'expérience.

Dans l'expérimentation qui suit, nous avons étudié le comportement de la réponse en fonction de l'épaisseur de la zone désertée. La source d'Am-Be a une émission neutronique égale à $2,8 \times 10^{6}$ neutrons . $\mathrm{s}^{-1}$ sur $4 \Pi$. L'équivalent de dose obtenu, après calcul, correspond à : $H^{\star}(10)=$ $1,19 \mathrm{mSv}$ [1].

Le convertisseur de polyéthylène utilisé a été choisi avec une épaisseur de $1 \mathrm{~mm}$ afin d'obtenir l'équilibre protonique, pour des neutrons d'énergie $10 \mathrm{MeV}$ et d'incidence normale. Les résultats obtenus sont résumés dans le tableau VI. II s'agit des valeurs moyennes correspondant à 5 mesures dans chaque cas. La plage d'énergie prise en compte est limitée par un seuil bas correspondant au canal 20 ( $E=390 \mathrm{keV}$ ).

TABLEAU VI

Réponses enregistrées et rapport signal/bruit lorsque l'épalsseur de la zone désertée de la diode varie (source Am-Be)

\begin{tabular}{|c|c|c|c|c|}
\hline$(\varphi \mathrm{m})$ & 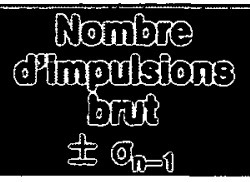 & 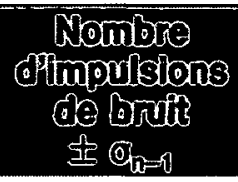 & $\begin{array}{l}\text { Aombro } \\
\text { offle } \\
\text { dimpulsions } \\
\text { f } \sigma_{n=0}\end{array}$ & $\begin{array}{l}\text { Bapport } \\
\text { signal sor } \\
\text { brut? }\end{array}$ \\
\hline $\begin{array}{l}80 \\
76 \\
710 \\
65 \\
60 \\
53 \\
45 \\
35\end{array}$ & 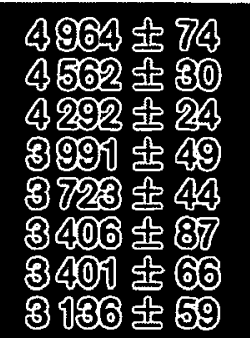 & 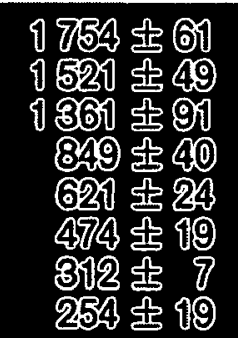 & 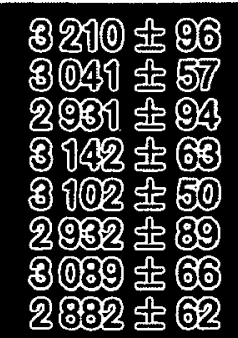 & $\begin{array}{r}1,83 \\
2,00 \\
2,05 \\
3,00 \\
5,00 \\
6,08 \\
9,40 \\
11,35\end{array}$ \\
\hline
\end{tabular}

Plusieurs conclusions s'imposent à la lecture du tableau VI. D'une part, pour une variation de la profondeur de zone désertée allant de 35 à $80 \mu \mathrm{m}$, la réponse ne varie pratiquement pas puisque l'on constate des valeurs extrêmes égales à 3210 et 2882 impulsions, pour une valeur moyenne de 3041 . Autrement dit, le spectre énergétique des protons issus du convertisseur et enregistrés par la diode se déforme (fig. 9 a et b) mais l'intégrale de ces spectres demeure dans les fluctuations statistiques. D'autre part, nous constatons que la réponse "parasite" due aux grandeurs interférentes varie comme la profondeur de zone désertée. Ces deux commentaires confirment les résultats et les discussions énoncés précédemment. Nous pouvons conclure en notant que la diminution de la profondeur de zone désertée permet une nette amélioration du rapport signal sur bruit. 

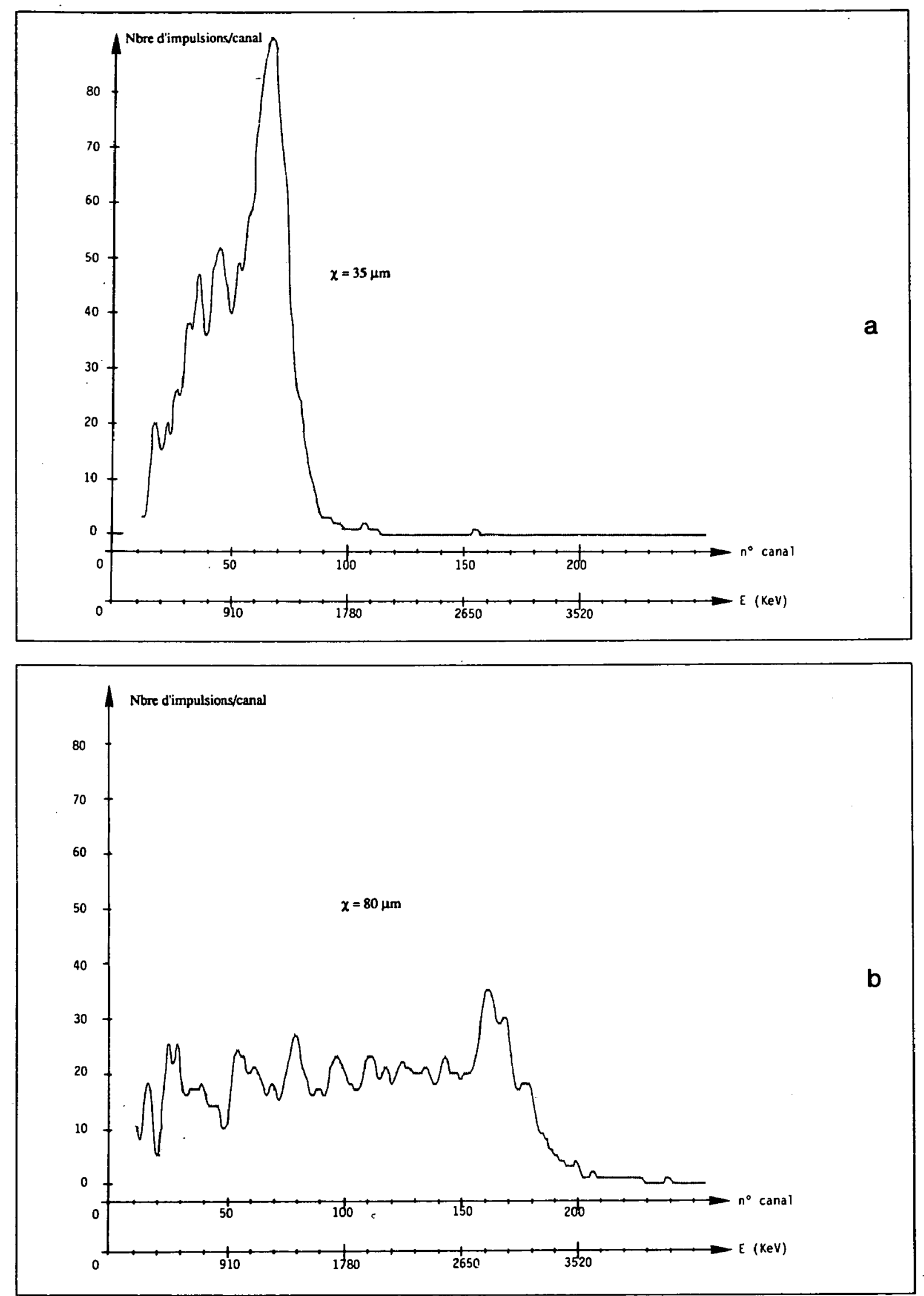

Fig. 9. - Modification du spectre énergétique en fonction de la profondeur de la zone désertée ; a : $\mathcal{X}=35 \mu \mathrm{m} ; \mathrm{b}: \mathcal{X}=80 \mu \mathrm{m}$. 


\section{VI.3. Expérimentation du dispositif grâce à une source étalon de neutrons thermiques}

Dans cette partie, nous utilisons des convertisseurs de polyéthylène d'épaisseur $150 \mu \mathrm{m}$ implantés avec des atomes de ${ }^{10} \mathrm{~B}$. L'épaisseur de la feuille de $\left(\mathrm{CH}_{2}\right)_{\mathrm{n}}$ et la densité d'atomes implantés sont variables afin de vérifier leur influence. La méthode différentielle a été réalisée sur une seule diode en effectuant deux mesures successives : la première de ces mesures avec un convertisseur implanté et la suivante avec un convertisseur non implanté. Ainsi, nous nous affranchissons, pour cette étape, de la dissymétrie existant au niveau des deux diodes, dissymétrie qui, sur le plan de la technologie de fabrication, devrait, pour la suite, être grandement réduite. Ce principe de mesure semble être difficilement applicable à un dispositif de routine.

Les convertisseurs ont été implantés avec 4 densités différentes d'atomes de ${ }^{10} \mathrm{~B}$ pour vérifier la linéarité entre densité d'atomes implantés et efficacité effective :

$-7,8 \times 10^{15}$ atomes $/ \mathrm{cm}^{2}$
$-6,5 \times 10^{15}$ atomes $/ \mathrm{cm}^{2}$
$-5,2 \times 10^{15}$ atomes $/ \mathrm{cm}^{2}$
$-3,9 \times 10^{15}$ atomes $/ \mathrm{cm}^{2}$

Compte tenu de l'énergie des particules $\alpha$ et des ions lithium et de leur ralentissement avant leur entrée dans la zone désertée, nous avons pu intégrer les spectres obtenus entre $560 \mathrm{keV}$ et 1,6 MeV, la contribution des particules citées n'intervenant pas en dehors de cette zone.

Les résultats obtenus sont regroupés dans le tableau VII qui fournit l'efficacité effective aux neutrons thermiques, en fonction de la densité d'implantation des convertisseurs.

TABLEAU VII

Résultats obtenus par des irradiations en neutrons thermiques sur "SIGMA"

à Cadarache, épaisseur de convertisseur de $\left(\mathrm{CH}_{2}\right)_{n}=150 \mu \mathrm{m}$; équivalent de dose $=0,18 \mathrm{mSv}$ )

\begin{tabular}{|c|c|c|c|c|}
\hline $\begin{array}{l}\text { Membre } \\
\text { de } \\
\text { mesures }\end{array}$ & $\begin{array}{c}\text { Complantafion } \\
\text { de }{ }^{40} \mathrm{~B} \\
\text { en atomes. } \mathrm{cm}^{-2}\end{array}$ & $\begin{array}{c}\text { Mombre } \\
\text { ditmpulsions } \\
\text { enreglstreces } \\
\text { \& } 0 \mathrm{n}=1\end{array}$ & 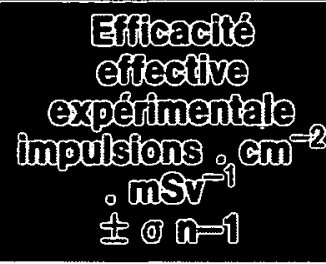 & 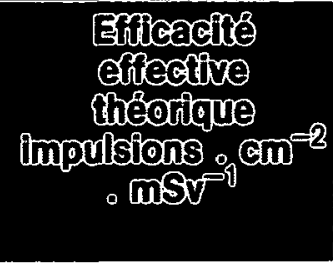 \\
\hline 6 & $7.8 \times 10^{15}$ & 7695 出 134 & 2850 卉 496 & 3000 \\
\hline 4 & $6.5 \times 10^{15}$ & 660,3 疋 170 & 2446 \& 630 & 2500 \\
\hline 4 & $5,2 \times 10^{15}$ & $502 \pm 210$ & $0859 \pm 778$ & 2000 \\
\hline 4 & $3,9 \times 1015$ & 383 \& 84 & 7418 \& 301 & 1500 \\
\hline
\end{tabular}


Ces résultats nous permettent d'affirmer que l'efficacité effective est bien une fonction linéaire de la densité d'implantation. Ils sont, en outre, en accord avec ceux que nous avions obtenus avec des DST [13].

\section{CONCLUSION}

A l'issue de ce travail, nous disposons d'un système électronique de dosimétrie neutronique dans une configuration que l'on peut qualifier de "macro-montage" qui, en l'état, n'est évidemment pas utilisable en dosimétrie individuelle. Par contre, grâce à cette configuration nous avons pu, dans un premier temps, montrer la faisabilité du système proposé qui repose sur deux éléments essentiels:

- le capteur constitué d'un convertisseur de polyéthylène implanté au bore 10 associé à un détecteur diode silicium (implanté au bore 11) qui recueille une réponse liée au nombre de particules chargées générées dans le convertisseur,

- les deux voies de mesures nécessaires pour réaliser la méthode différentielle qui permet de limiter de façon importante les signaux perturbateurs dus aux grandeurs d'entrée interférentes et au bruit électronique.

Dans une deuxième étape, nous avons effectué des tests systématiques du dispositif dans des champs de neutrons rapides et thermiques. De l'ensemble de ces mesures on peut tirer des premières conclusions et des enseignements utiles à la mise au point définitive du dosimètre personnel que l'on peut envisager raisonnablement aujourd'hui. On retiendra principalement ici :

1 - La meilleure qualité du dosimètre électronique comparé au dosimètre équivalent constitué d'un détecteur CR 39. Cela se traduit à trois niveaux :

- une efficacité effective qui double (à convertisseur identique) pour les neutrons rapides; cela est dû au fait que les limitations énergétiques et/ou angulaires sont relativement faibles, et donc que l'efficacité intrinsèque théorique du convertisseur est comparable à l'efficacité effective expérimentale du capteur;

- malgré l'intrusion des grandeurs interférentes, les études statistiques font apparaître une nette amélioration de l'erreur quadratique moyenne; ainsi pour un équivalent de $5 \mathrm{mSv}$ en neutrons rapides $(3,4 \mathrm{MeV}$ ) on obtient (convertisseur de $35 \mu \mathrm{m}$ ) pour le dosimètre à DST : $2481 \pm 327$ traces. $\mathrm{cm}^{-2}$ et pour le dosimètre électronique : $4329 \pm 102$ impulsions $\mathrm{cm}^{-2}$;

- une mesure de l'équivalent de dose, sinon instantanée du moins au bout d'un temps relativement court.

2 - La possibilité de travailler avec un débit de dose plus faible avec notre système qu'avec celui proposé par Eisen [6] qui fixe un seuil bas de $3 \mathrm{mSv} . \mathrm{h}^{-1}$, alors que nous pouvons atteindre une centaine de microsieverts par heure. 
3 - La limitation principale qui intervient relève d'une étude systématique nécessaire pour réduire l'influence des grandeurs interférentes et du bruit électronique et ce, malgré l'application de la méthode différentielle. L'intervention du rayonnement $y$ ainsi que celle des interactions directes "neutrons silicium" sont importantes. En dessous de $400 \mathrm{keV}$, celle du rayonnement $\gamma$ est prépondérante. Dans ces conditions, toutes les impulsions d'énergie inférieure à $400 \mathrm{keV}$ ne sont pas prises en compte dans le calcul de l'équivalent de dose. Grâce à la méthode différentielle, une légère amélioration est apportée par rapport au dispositif électronique d'Eisen dont le niveau de coupure est fixé à $700 \mathrm{keV}$.

4 - La méthode que nous proposons (existence d'un seuil à $400 \mathrm{keV}$ ), doit aussi permettre une première discrimination $y$ - neutrons dans les champs mixtes et mériterait d'être testée dans des champs bien connus. II ne fait pas de doute que notre structure mérite une évolution qui devrait aboutir à une discrimination précise de l'équivalent de dose $\gamma$ de celui des neutrons. II s'agit là d'un projet qui nécessite des études supplémentaires dont le résultat pourrait constituer une amélioration indéniable du présent système.

5 - La réalisation d'un double détecteur, permettant d'assurer une meilleure symétrie des deux voies, donc d'accroître les performances de la méthode différentielle, doit faire l'objet d'attentions particulières, mais il semble très probable que ses qualités puissent être améliorées sensiblement.

D'autres investigations sont encore nécessaires, mais déjà des points positifs permettent d'envisager l'étape de miniaturisation du système à l'issue de laquelle des tests "grandeur nature" devraient être menés sur différents sites dans des champs mixtes, en parallèle avec les dosimètres actuellement utilisés.

\section{REMERCIEMENTS}

Ce travail a pu être réalisé grâce aux soutiens de la SFRP et du CEA, Service d'instrumentation et de dosimétrie en radioprotection (SIDR), de Fontenay-aux-Roses et de Cadarache (SIDR). Qu'ils trouvent ici, tous nos remerciements.

B. BARELAUD a été boursier de la SFRP pendant la période du $1^{\text {er }}$ octobre 1987 au 30 septembre 1988.

\section{BIBLIOGRAPHIE}

[1] AFNOR. - Rayonnements neutroniques de référence destinés à l'étalonnage des instruments de mesure des neutrons utilisés en radioprotection et à la détermination de leur réponse en fonction de l'énergie des neutrons. Norme NF M-60-516, ISO-8529, 1989. 
[2] DECOSSAS J.L. - Endommagements des dérivés cellulosiques par des ions. Modélisation de la trace. Détection des protons. Application à la dosimètrie neutronique. Thèse $n^{\circ}$ 12-82, Université de Limoges, 1988.

[3] DECOSSAS J.L., SADAKA S., VAREILLE J.C., MAKOVICKA L., TEYSSIER J.L. Theoretical study of the contribution of a radiator to the sensitivity of a rapid neutron dosemeter. Nucl. Tracks Radiat. Meas., 1986, 12, 543-546.

[4] DECOSSAS J.L., VAREILLE J.C., MOLITON J.P., TEYSSIER J.L. - Theoretical study of response of a rapid neutron dosemeter based on track detector. Radiat. Prot. Dosim., 1983, 5, 163-170.

[5] EISEN Y., ENGLER G., OVADIA E., SHAMAI Y., BAUM Z., LEVY Y. - A small size neutron and gamma dosimeter with a single silicon surface barrier detector. In : DOE workshop on personnal neutron dosimetry, Acapulco, Mexico, PNL-SA-12352 1983, 157-175.

[6] EISEN Y., ENGLER G., OVADIA E., SHAMAI Y. - A combined real time wide energy range neutron dosimeter and survey meter from high neutron dose rates with Si surface barrier detectors. Nucl. Instrum. Methods 1983, A 211, 171-178.

[7] GIBSON J.A.B. - Individual neutron dosimetry. Radiat. Prot. Dosim., 1988, 23 , 109-115.

[8] GIBSON J.A.B. - A neutron dosimetry system for use in processing plants. In : Advances in radiation protection monitoring, Stockholm, 1978. Vienna: AIEA, 1979, 197-206.

[9] HEINZELMANN - Neutron dosimetry. Report for the project group on neutron dosimetry of the CEC. EUR-5484, 1976, 91-95.

[10] KEMMER J., BURGER P., HENCK R., HEIJNE E. - Performance and applications of passivated ion-implanted silicon detectors. IEEE Trans. Nucl. Sci., 1982, 29, 733-737.

[11] KEMMER J. - Fabrication of low noise silicon radiation detectors by planar process. Nucl. Instrum. Methods 1980, A 169, 499-502.

[12] KNOLL G.F. - Radiation detection and measurements. New-York : J. Wiley, 1979, 551-596.

[13] MAKOVICKA L. - Contribution à la dosimétrie neutron-gamma. Etude d'un ensemble radiateur-détecteur type CR 39. Thèse $n^{\circ} 17-87$, Université de Limoges, 1987.

[14] MAKOVICKA L., BARELAUD B., DECOSSAS J.L., VAREILLE J.C. - Detection of thermal neutron by CR 39 using a boron implanted convertor. Radiat. Prot. Dosim., 1988, 23 (1/4) 191-194.

[15] MAKOVICKA L., DECOSSAS J.L., VAREILLE J.C. - Experimental study of dosimetry efficiency of a radiator CR 39 fast neutron dosemeter. Radiat. Prot. Dosim., 1987, 20 (1/2) 63-66.

[16] MAKOVICKA L., SADAKA S., VAREILLE J.C., DECOSSAS J.L., TEYSSIER J.L. Study of a polyethylene CR 39 fast neutron dosemeter, Part I: Characteristics of proton fluxes emitted by a polyethylene radiator. Radiat. Prot. Dosim., 1987, 16 (4) 273-279. 
[17] MESSENGER G.C., ASH M.S. - The effects of radiation on electronic systems. New-York: Van Nostrand Reinhold Co, 1987, 369-395.

[18] PARATTE P.A., ROBERT P. - Système de mesure. Paris: Dunod, 1986.

[19] PAUL D. - Dosimétrie neutronique par ensemble "convertisseur - CR 39". Comptage automatique des traces. Etude du bruit de fond. DEA de Physique Radiologique, Université de Toulouse, 1988.

[20] QUAM W., DEL DUCA T., PLAKE T., GRAVES W., DEVORE T. - Pocket neutron rem meter. CONF-811012-63, 1981, 637-640.

[21] SADAKA S. - Etudes théoriques et expérimentales d'un dosimètre pour les neutrons rapides. Thèse $n^{\circ} 30-83$, Université de Toulouse, 1984.

[22] SADAKA S., MAKOVICKA L., VAREILLE J.C., DECOSSAS J.L., TEYSSIER J.L. Study of a polyethylene CR 39 fast neutron dosemeter, Part II : Dosimetry efficiency of the device. Radiat. Prot. Dosim., 1987, 16 (4) 281-287.

[23] SADAKA S., MAKOVICKA L., VAREILLE J.C., DECOSSAS J.L. - Conribution of heavy recoils to the response of a neutron dosemeter. Radiat. Prot. Dosim., 1987, 18 (4) 215-220.

[24] TUBIANA M., DUTREIX J., DUTREIX R., JOCKEY P. - Bases physiques de la radiothérapie et de la radiobiologie Paris: Masson, 1963.

[25] TYREE W.H., FALK R.B. - A personnel neutron dosemeter. In : 9th. DOE workshop on neutron personnal dosimetry, Las Vegas, Nevada, PNLSA-10714 1981, 154-161.

[26] VAREILLE J.C., SADAKA S., DECOSSAS J.L., TEYSSIER J.L. - Experimental response of a dosemeter ( $\mathrm{rad} / \mathrm{CR}$ 39) for neutrons in the energy range $100 \mathrm{keV}-3 \mathrm{MeV}$. Nucl. Tracks Radiat. Meas., 1986, 12, 589-592.

[27] VAREILLE J.C., DECOSSAS J.L., SADAKA S., TEYSSIER J.L. - CR 39 used as detector neutron dosemeter. Nucl. Intrum. Methods 1986, A 17 (3) 280-282. 